\title{
Prevalence of primary infertility among selected group of Sudanese women with infertility disorders
}

\begin{abstract}
Background: Primary infertility is a health issue among women over the world.

Justification: There is inadequate data about the percentage of women with primary infertility disorder attended to infertility and assisted reproduction centers nationally and globally.
\end{abstract}

Objectives: To recognize the prevalence of primary infertility among Sudanese women with infertility disorders attended to Banoon Center of obstetrics and gynecology and assisted reproduction, Khartoum, Sudan.

Material and Method: Hospital based -descriptive cross sectional study, 143 Sudanese women attended to Banoon center of obstetrics and gynecology and assisted reproduction were involved in the study.

Result: The primary infertile women between the participants were $60.84 \%$.

Conclusion: Additional studies must be done with large sample size and at different infertility health care centers

Keywords: prevalence, primary infertility, women, Sudan
Volume 8 Issue I - 2019

\author{
Mohammed Omer Mohammed Hussein,' \\ Mohammed A Gafoor A Gadir,' Mosab \\ Nouraldein Mohammed Hamad ${ }^{2}$ \\ 'Department of obstetrics and gynecology and reproduction, \\ Banoon center, Sudan \\ ${ }^{2}$ Department of health Sciences, Elsheikh Abdallah Elbadri \\ University, Sudan
}

Correspondence: Mosab Nouraldein Mohammed Hamad, Department of Medical Parasitology, Faculty of Health Sciences Elsheikh Abdallah Elbadri University, Sudan,

Email musab.noor13@gmail.com

Received: February 07, 2019 | Published: February 26, 2019

\section{Introduction}

Infertility is when a couple can't get pregnant (conceive) despite having regular unprotected sex. ${ }^{1}$ The inability to have children influences men and women across the globe. Infertility can cause distress and depression, as well as discrimination and ostracism. ${ }^{2}$

Universal infertility prevalence rates are difficult to determine, because of the presence of both male and female factors which complicate any approximation which may only address the woman and an outcome of a pregnancy diagnosis or live birth. ${ }^{3}$

Primary infertility define as that the pair has never conceived. Secondary infertility means that the pair has experienced a pregnancy before and failed to conceive later. worldwide, most infertile couples suffer from primary infertility. ${ }^{4}$ Most cases of women infertility are attributed to problems with ovulation. Without ovulation, there are no ova to be fertilized. Some signs that a female is not ovulating normally include irregular or absent menstrual periods. ${ }^{5}$

Ovulation disorders are often caused by polycystic ovarian syndrome (PCOS). PCOS is a hormone disturbance condition which can influence with normal ovulation. PCOS is the most common reason of female infertility. Primary ovarian insufficiency (POI) is a further cause of ovulation problems. POI exists when a woman's ovaries stop working normally before she is forty. ${ }^{5}$ The specific reason of PCOS is unknown. Early investigation and cure along with weight loss may decrease the danger of long-term consequences such as type 2 diabetes and cardiac disease. ${ }^{6}$

Primary infertility is low and not over $3 \%$ in less than a third of the 28 African countries analyzed. ${ }^{7}$ Infertility is real problems affecting many of Sudanese women and it may lead to many social and economical complications which may affect the community as a whole. Also the infertility health care centers are not equally distributed throughout the country. Prevalence of poverty and high cost of infertility investigating and treating tools need the support of the charity organizations to support the heath care professionals in order to reach the majority of infertile women throughout the country and to solve this health and socioeconomic trouble.

\section{Justification}

There is scarce data about the percentage of women with primary infertility disorder attended to infertility and assisted reproduction centers nationally and globally.

\section{Objectives}

To know the prevalence of primary infertility among Sudanese women with infertility disorders attended to Banoon Center of obstetrics and gynecology and assisted reproduction, Khartoum, Sudan.

\section{Material and method}

\section{Study design}

Hospital based- descriptive cross sectional study

\section{Study area}

Banoon Center of obstetrics and gynecology and assisted reproduction, Khartoum, Sudan

\section{Study period}

March to October 2017

\section{Study population}

Sudanese women with infertility disorders attended to Banoon center during the study period.

\section{Sample size}

143 Sudanese women with infertility disorder

\section{Data collection}

Well constructed questionnaire was formed and filled by each participant. 


\section{Ethical approval}

Every participant was informed about the aim of the study and each one was consent to be involved in it.

\section{Result}

The primary infertile women between the participants were $60.84 \%$.

\section{Conclusion}

Further studies must be done with large sample size and at different infertility health care centers.

\section{Acknowledgments}

None.

\section{Conflicts of interest}

The author declares there are no conflicts of interest.

\section{References}

1. https://www.nhs.uk/conditions/infertility/

2. Mascarenhas MN, Flaxman SR, Boerma T, Vanderpoel S, et al. National, regional, and global trends in infertility prevalence since 1990: a systematic analysis of 277 health surveys. PLoS Med. 2012;9(12):e1001356.

3. WHO. Global prevalence of infertility, infecundity and childlessness. UK: 2012. 12 p.

4. https://www.aimu.us/2018/01/10/infertility-symptoms-causesdiagnosis-management-and-prevention/

5. https://www.womenshealth.gov/a-z-topics/infertility

6. https://www.mayoclinic.org/diseases-conditions/pcos/symptomscauses/syc-20353439

7. https://www.ncbi.nlm.nih.gov/pubmed/10817127 